\title{
Bacia hidrográfica do Rio Roosevelt: um território fluvial integrador na Pan-Amazônia
}

Por meio da caracterização de uma Bacia Hidrográfica é possível identificar sua situação atual e vislumbrar as tendências evolutivas, contribuindo para melhor gestão e regulação dos usos que ocorrem nesta bacia, de forma a conservar os recursos hídricos em quantidade e qualidade e proporcionar melhorias para sociedade local, assim sendo esta pesquisa tem como objetivo identificar a estrutura socioterritorial dos municípios pertencentes à Bacia Hidrográfica do Rio Roosevelt, um território integrador de três Estados da Pan-Amazônia, a luz do método PEIR. Para a determinação do cenário econômico, ambiental e social da Bacia Hidrográfica os indicadores foram extraídos a partir de dados secundários disponibilizados pelo Instituto Brasileiro de Geografia e Estatística - IBGE a partir do sistema agregador de informações Cidades@. Os indicadores considerados para o panorama socioeconômico e ambiental consistiram nos dados de População, Densidade Demográfica, Índice de Desenvolvimento Humano - IDH, taxa de escolarização de 6 a 14 anos de idade, Índice de Desenvolvimento da Educação Básica - IDEB dos anos População, Densidade Demográfica, Índice de Desenvolvimento Humano - IDH, taxa de escolarização de 6 a 14 anos de idade, Índice de Desenvolvimento da Educação Básica - IDEB dos anos iniciais e finais do ensino fundamental da rede pública, Salário médio mensal de trabalhadores formais (Salário mínimo), proporção de estabelecimentos envolvida na produção de Lavouras, Pastagens, Matas e Florestas, Produto Interno Bruto - PIB, casos de internações por Diarreia por mil habitantes, taxa de mortalidade infantil e porcentagem de domicílios com Esgotament Sanitário adequado. Os dados foratisa anilidade Pressão-Estado-Impacto-Resposta (PEIR) desenvolvida de Análise Estatística (RAE) e aplicação dos indicadores de pressão, estado e impacto e Programa das Nações Unidas e Meio Ambiente - PNUMA. Os dados foram organizados em gráficos e tabelas, por meio do software Excel, versão 2016. A partir dos dados analisados constatouse que os municípios de Vilhena e Cacoal se destacam com valores altos de IDH $(0,731$ e 0,718), por outro lado em Novo Aripuanã foi observado um valor de IDH baixo (0,554), o menor da indicadores estudados revelam que há diferentes pressões no decorer apresentadas pelo território em que abrange e na qualidade ambiental e de vida.

\section{Watershed of the Roosevelt River: an integrating river territory in the Pan-Amazon}

The Hydrographic Basin characterization enable its current situation identification and glimpse its evolutionary trends, contributing to better management and regulation of the basin uses, in order to conserve water resources in quantity and quality and provide improvements for local society. Therefore, this research aims to identify the Roosevelt River Basin municipalities socio-territorial structure, an integrating territory of three Pan-Amazon States, in the light of DPSIR method. To determine the economic, environmental and social Hydrographic Basin scenario the indicators were extracted from secondary data provided by the Brazilian Institute of Geography and Statistics - IBGE from the information aggregator system Cidades@. The socioeconomi and environmental scenario indicators consisted of data on Population, Demographic Density, Human Development Index -HDI, School Attendance Rate of 6 to 14 years old children, Basic Education Development Index - IDEB from public elementary school initial and final years, formal workers Average Monthly Wage (minimum wage), proportion of establishments involved in the production of Crops, Pastures, Bushes and Forests, Gross Domestic Product - GDP, hospitalizations of Diarrhea Cases per thousand inhabitants, Infant Mortality Rate and percentage of households with a Programme - UNEP. The data were organized in graphics and the us with high HDI values ( 0.731 and 0.718$)$, on the other hand in Novo Aripuanã a low HDI value $(0.554)$ was observed, the lowest in the Basin, these results may have a direct influence of economic, education and health indicators, which showed more satisfactory values in the first two municipalities. As a result, the studied indicators reveal different pressures throughout the Basin, mainly related to economic and environmental factors, which can influence the territory characteristics and the environmental and life quality.

Keywords: SAR; DPSIR; Socioterritorial.

Topic: Desenvolvimento, Sustentabilidade e Meio Ambiente

Reviewed anonymously in the process of blind peer

Naara Ferreira Carvalho de Souza (iD)

Fundação Universidade Federal de Rondônia, Brasil http://lattes.cnpq.br/9254969219816395

http://orcid.org/0000-0002-6407-9999

naaraferreira94@gmail.com

Caryne Ferreira Ramos (ic

Fundação Universidade Federal de Rondônia, Brasil http://lattes.cnpq.br/8471241504526836

http://orcid.org/0000-0003-1562-2251

caryne.framos@gmail.com

Douglas Linz Ricardo (iD

Fundação Universidade Federal de Rondônia, Brasil

http://lattes.cnpq.br/2845758354231821

http://orcid.org/0000-0002-2328-4910

douglas.linz@gmail.com

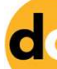

DOI: 10.6008/CBPC2318-2881.2021.001.0015
Received: 04/12/2020

Approved: 21/02/2021
Rafaela Carvalho Barcelos (iD)

Fundação Universidade Federal de Rondônia, Brasil

http://lattes.cnpq.br/0379074607707850

http://orcid.org/0000-0002-5466-3737

eng.rafaelacbarcelos@gmail.com

\section{Nubia Caramello}

Fundação Universidade Federal de Rondônia, Brasil

http://lattes.cnpq.br/8155132371455051

http://orcid.org/0000-0002-2167-9759

geocaramellofrj@gmail.com

\section{Referencing this:}

SOUZA, N. F. C.; RAMOS, C. F.; RICARDO, D. L.; BARCELOS, R. C.; CARAMELLO, N.. Bacia hidrográfica do Rio Roosevelt: um território fluvial integrador na Pan-Amazônia. Nature and Conservation, v.14, n.1, p.129-140, 2021. DOI: http://doi.org/10.6008/CBPC23182881.2021.001.0015 


\section{INTRODUÇÃO}

A partir da implantação da Lei 9.433/97, nasce as unidades de gestão hidrográfica por meio da implantação de Comitês de Bacia Hidrográfica, formando o que podemos classificar a partir de Cotta et al. (2019) como territórios fluviais e por Nunes et al. (2016) e Barreto (2019) como territórios de Águas.

O estado de Rondônia, em 2014, sanciona por meio de cinco Decretos as Bacias Hidrográficas que poderiam implantar os seus Comitês Hidrográficos, entretanto a Bacia do Rio Roosevelt, não integrou a série de Comitês de Bacia criados. Sendo uma Bacia com presença de conflitos diretamente por razões minerais, seja o diamante sólido ou o líquido como é chamada a água, vem sendo impactada pelo extrativismo depredador, que não segue nenhuma norma de proteção da biodiversidade e é realizado clandestinamente, impactando diretamente o corpo hídrico desse território fluvial (CARAMELLO et al., 2016).

A bacia hidrográfica se converte em uma unidade de gestão de territórios fluviais, demarcados por interesses dos atores hidrográficos, por ser considerada um ente sistêmico sendo realizada nela os balanços de entrada através das precipitações e saída no seu exutório (PORTO et al., 2008). A caracterização da bacia apresenta os principais aspectos socioambientais que possibilitam uma visão abrangente dos principais usos dos recursos hídricos e do solo, ao qual possibilita a identificação da situação atual, assim como as suas tendências evolutivas.

Dessa forma adota-se o método Pressão-Estado-Impacto-Resposta (PEIR), que analisa as intervenções antrópicas em um território por meio de indicadores, e vem sendo frequentemente utilizado para estudos socioambientais urbanos, como também aplicado em análises de territórios fluviais (CARAMELLO et al., 2011; COTTA et al., 2019), tem como objetivo a sensibilização sobre questões ambientais, proporcionando opções para ações, através das quais se torna possível dentre outras atribuições, fazer análise de medidas corretivas, adotar novas alternativas de enfrentamento dos problemas ambientais assim como identificar competências e níveis de responsabilidade dos agentes e atores sociais comprometidos (SILVA et al., 2020).

Desta forma, para a identificação e aplicação do método (PEIR) das potencialidades da bacia serão analisados os indicadores: população, Densidade Demográfica, Índice de Desenvolvimento Humano - IDH, taxa de escolarização de 6 a 14 anos de idade, IDEB dos anos iniciais e finais do ensino fundamental da rede pública, salário médio mensal de trabalhadores formais (salário mínimo), produção de Lavouras, Pastagens, Matas e Florestas, PIB, casos de internações por diarreia, taxa de mortalidade infantil e porcentagem de domicílios com Esgotamento Sanitário adequado.

Esses indicadores serão organizados e analisados por meio do Relatório de Análise Estatística (RAE). Sendo assim, o objetivo do estudo é identificar a estrutura socioterritorial dos municípios pertencentes à Bacia Hidrográfica do Rio Roosevelt, um território integrador de três Estados da Pan Amazônia, a luz do método PEIR. 


\section{MATERIAIS E MÉTODOS}

\section{Área de estudo}

A bacia hidrográfica do Rio Roosevelt, apresentada na Figura 1, está localizada na Região Norte do Brasil e delimitada entre os paralelos 70 34' e 12ㅇ 40' de latitude Sul e os meridianos de 59o 27' e 61은 40 de longitude Oeste, abrangendo áreas dos estados de Rondônia, Mato Grosso e Amazonas.

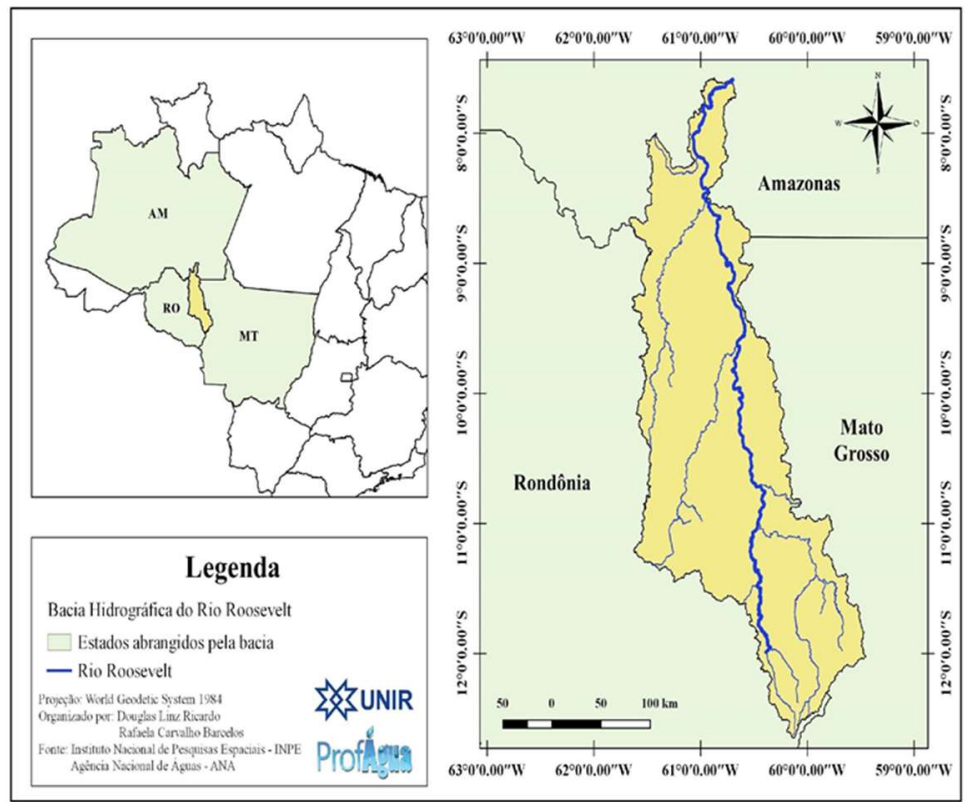

Figura 1: Localização e delimitação da bacia hidrográfica do Rio Roosevelt.

A área drenada pela bacia corresponde a aproximadamente $60.039,65 \mathrm{~km}^{2}$, onde cerca de $60 \%$ desta se encontra localizada no estado do Mato Grosso, compreendendo parte das áreas dos municípios de Rondolândia, Colniza, Aripuanã e Juína. Em Rondônia a área drenada abrange parte dos municípios de Vilhena, Pimenta Bueno, Espigão do Oeste, Cacoal e Ministro Andreazza. A menor porção da bacia está localizada no município de Novo Aripuanã no estado do Amazonas, onde se encontra o exutório da bacia.

Essa Bacia Hidrográfica tem como curso d'água principal o Rio Roosevelt, conhecido também como Rio da Dúvida, descoberto em 1909 pela Comissão Rondon. Trata-se de um afluente da margem esquerda do Rio Aripuanã, estando sua nascente localizada na Chapada dos Parecis, nas proximidades da sede municipal de Vilhena - RO. Os principais tributários do Rio Roosevelt são os rios Madeirinha e Branco pela margem esquerda e Capitão Cardoso pela margem direita (RIBEIRO, 2017).

Conforme a Lei Complementar no 255 de 25 de janeiro de 2002 do Estado de Rondônia em consonância com a Lei no 9.433 de 08 de janeiro de 1997 que instituiu a Política Nacional de Recursos Hídricos, a Bacia Hidrográfica é definida como uma unidade territorial, assim sua delimitação se dá através de divisores físicos (topográficos) os quais podem extrapolar os limites políticos de municípios e estados, o que é o caso desta bacia. Assim, a área de estudo da presente pesquisa compreende todos os municípios abrangidos pela área de drenagem da bacia do Rio Roosevelt (RONDÔNIA, 2002).

Para a determinação do cenário econômico, ambiental e social da Bacia Hidrográfica os indicadores foram extraídos a partir de dados secundários disponibilizados pelo Instituto Brasileiro de Geografia e 
Estatística - IBGE a partir do sistema agregador de informações Cidades@, cujo acesso se dá através do endereço eletrônico. ${ }^{1}$

Os indicadores considerados para o panorama socioeconômico e ambiental da bacia consistiram nos dados de população, Densidade Demográfica, IDH, taxa de escolarização de 6 a 14 anos de idade, IDEB dos anos iniciais e finais do ensino fundamental da rede pública, salário médio mensal de trabalhadores formais (salário mínimo), produção de Lavouras, Pastagens, Matas e Florestas, PIB, casos de internações por diarreia, taxa de mortalidade infantil e porcentagem de domicílios com Esgotamento Sanitário adequado, disponíveis no sistema do IBGE para cada um dos municípios inseridos na bacia hidrográfica. Seguramente outros dados tem igual valor para análise, entretanto visando atender ao objetivo proposto foram minuciosamente decididos pelos indicadores supracitados.

Os dados obtidos no sistema foram analisados de forma direta a partir da metodologia de estruturação de Relatório de Análise Estatística (RAE) (NOLAN et al., 2000) e aplicação dos indicadores de pressão, estado e impacto da matriz de indicadores de sustentabilidade Pressão-Estado-Impacto-Resposta (PEIR) (CARAMELLO et al., 2011; SILVA et al., 2012) desenvolvida pela Organização para a Cooperação e o Desenvolvimento Econômico - OCDE e pelo Programa das Nações Unidas e Meio Ambiente - PNUMA (2002). A tabulação dos dados se deu à por meio do software Excel (versão 2016), onde se procedeu com as análises estatísticas e produção de gráficos e tabelas.

\section{RESULTADOS E DISCUSSÃO}

Com a análise dos indicadores extraídos do Banco de Dados do IBGE - Cidades@, observou-se que a população total nos municípios da bacia no ano de 2010 era de cerca de 337.026 habitantes, e para o ano de 2020 a estimativa é de 400.997 habitantes, um percentual de aumento populacional de cerca de 19,98\% em dez anos.

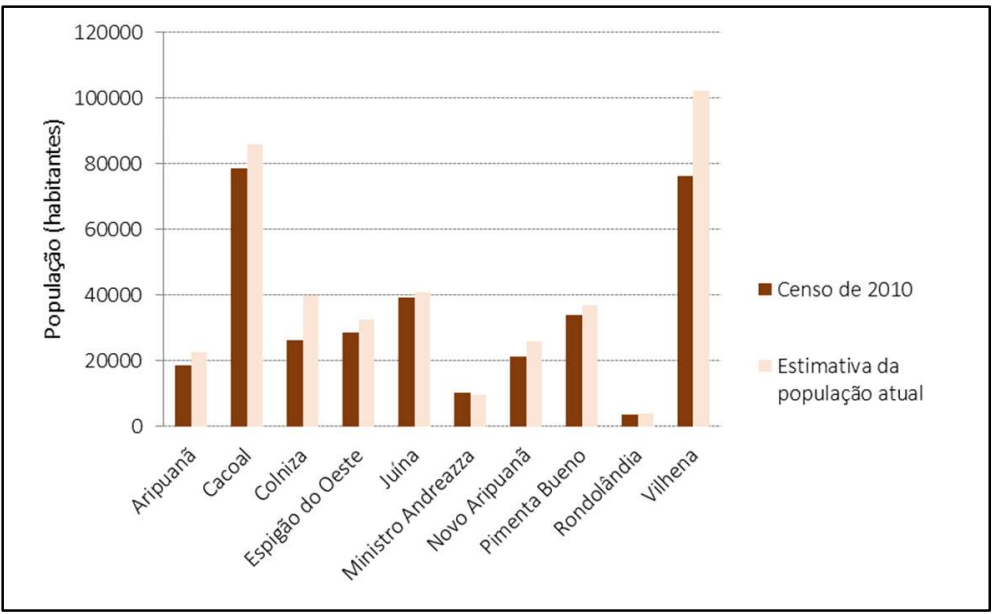

Figura 2: População dos municípios pertencentes à bacia Hidrográfica do rio Roosevelt, no censo de 2010, e estimativa para 2020.

Os municípios com maior população da Bacia do Rio Roosevelt, conforme observado na Figura 2, são

\footnotetext{
${ }^{1}$ http://cidades.ibge.gov.br
} 
Vilhena e Cacoal, já as maiores estimativas de aumento populacional, de 2010 para 2020, são observadas nos municípios de Colniza e Vilhena, crescendo a um percentual de 51,09 e 34,13\%, respectivamente. Dos dez municípios analisados, em nove ocorreu um aumento da população, por outro lado em Ministro Andreazza há uma estimativa de redução a um percentual de 7,67 \%.

O crescimento populacional indica as pressões, crescentes ou decrescentes, exercidas no meio ao longo do tempo, o que gera impactos - como a contaminação da água e solo, e a poluição atmosférica por Indústrias e Automóveis - decorrentes do uso desordenado dos recursos naturais (PNUMA, 2002).

Sendo assim, o aumento da população no município de Vilhena pode estar relacionado à instalação de indústrias e fábricas nos últimos anos, a exemplo dos frigoríficos, que atraem mão de obra para a região, e até mesmo em decorrência do avanço da agricultura, já que de acordo com Silva (2013) o município se destaca na produção de arroz, soja e milho, sendo Vilhena o maior produtor de soja do estado (IDARON, 2018).

A densidade demográfica é um indicador de estado, corresponde ao número de habitantes por quilômetro quadrado de uma região (hab./ $\mathrm{km}^{2}$ ), evidenciado uma pressão de uso de um território, ampliando quando este não segue as normativas ambientais. Em áreas com intenso povoamento e urbanização, também há maior pressão sobre o ambiente e consequentemente uma maior degradação, além da ocorrência de outros impactos de origem social e econômica (SANTOS et al., 2018).

O município que possui maior densidade demográfica é Cacoal, com 20,72 hab.km², seguido de Ministro Andreazza, com 12,97 hab.km², que apesar de ter uma das menores populações apresentou a segunda maior densidade demográfica da bacia (Tabela 1).

Tabela 1: Densidade demográfica (hab. $\mathrm{km}^{2}$ ) por município, no ano de 2010.

\begin{tabular}{ll}
\hline Municípios & Densidade Demográfica (hab. $\left.\mathrm{km}^{2}\right)$ \\
\hline Aripuanã & 0,74 \\
Cacoal & 20,72 \\
Colniza & 0,94 \\
Espigão do Oeste & 6,36 \\
Juína & 1,5 \\
Ministro Andreazza & 12,97 \\
Novo Aripuanã & 0,52 \\
Pimenta Bueno & 5,42 \\
Rondolândia & 0,28 \\
Vilhena & 6,62 \\
\hline
\end{tabular}

Fonte: IBGE (2010).

Os municípios de Vilhena e Cacoal apresentaram IDHs altos $(0,731$ e 0,718$)$, os maiores da bacia como observado na Figura 3, o que pode estar ligado ao fato do PIB (Figura 8) está entre os mais altos, assim como os indicadores de acesso à educação e saúde demonstrarem, em sua maioria, valores satisfatórios (Figuras 4 e 5) se comparado a outros municípios.

O IDH como um indicador de estado, conforme destacado pelo Programa das Nações Unidas - PNUD, evidencia o desenvolvimento de uma população (com valores de 0 a 1), através dos seguintes critérios: saúde, educação e renda (PNUD et al., 2013). Esse indicador "reflete a estreita relação com os debates em torno da mensuração da qualidade de vida. A rigor, um indicador sobre esse tema se baseia na admissão de que a 
qualidade de vida não se resume à esfera econômica da experiência humana" (SCARPIN et al., 2007).

A taxa de escolarização está relacionada à porcentagem de indivíduos, em determinada faixa etária, nesse caso de 6 a 14 anos, que está frequentando a escola em comparação à quantidade total de pessoas nessa mesma faixa etária (IBGE, 2020). A partir da Figura 4, como indicador de educação, é possível constatar que as maiores taxas de escolarização de 6 a 14 anos de idade, se aproximando de 100\%, estão em Vilhena (97,8\%), Pimenta Bueno $(97,7 \%)$ e Cacoal $(97,6 \%)$ (Figura 4). Por outro lado, no município de Novo Aripuanã é observada a menor taxa de escolarização de alunos nessa faixa etária (88\%), o único que possui taxa inferior a $90 \%$.

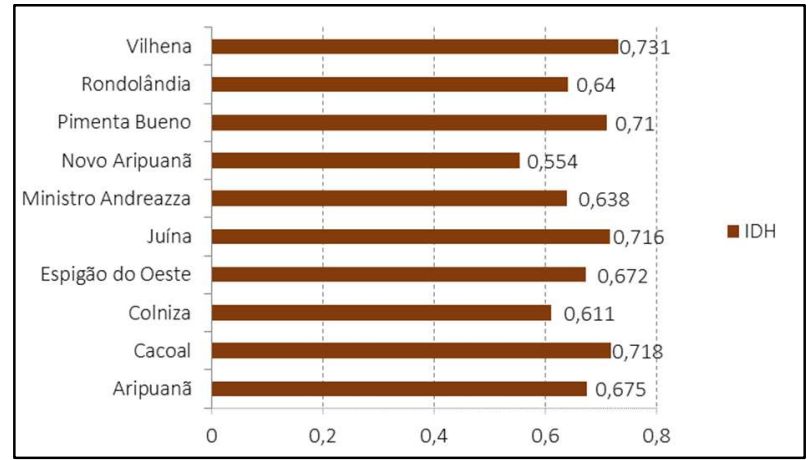

Figura 3: IDH dos municípios pertencentes à Bacia Hidrográfica do Rio Roosevelt, no ano 2010.

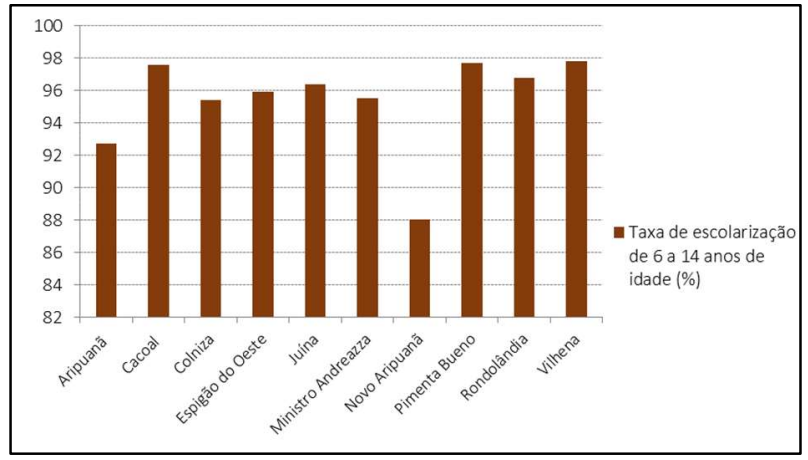

Figura 4: Taxa de escolarização de 6 a 14 anos de idade (\%) dos municípios pertencentes à Bacia Hidrográfica do Rio Roosevelt, no ano 2010

No Brasil, no ano de 2010, 96,7\% das pessoas na faixa etária de 6 a 14 anos frequentavam a escola, cerca de 3,3\% (o equivalente a 966 mil pessoas) estavam fora dela (IBGE, 2010). Os resultados obtidos na Bacia do Rio Roosevelt se aproximaram da taxa nacional, na maioria dos municípios, apenas em Novo Aripuanã e Aripuanã os valores ficaram distantes.

Neste contexto, de acordo com o Fundo das Nações Unidas - UNICEF (2014), apesar do ensino fundamental não ser a faixa etária com os maiores índices de indivíduos fora da escola no país, assim como em outras séries esse fato está relacionado à desigualdade social. As crianças e adolescentes negros, quilombolas, indígenas, com problemas na justiça, que residem em área rural ou vindos de famílias com baixa escolaridade são os grupos mais excluídos.

O IDEB é um indicador de estado que foi criado pelo Ministério da Educação - MEC, no ano de 2007, com intuito de avaliar a aprendizagem e a qualidade do ensino oferecido nas várias regiões do país e assim propor metas para o avanço do ensino a nível nacional, estadual e municipal. É um índice medido a cada dois anos e tem por objetivo o alcance da média de 6 pontos até o ano de 2022, utilizando dois fatores de cálculo: o rendimento escolar e os resultados de desempenho obtidos em avaliações realizadas pelo Instituto Nacional de Estudos e Pesquisas - INEP (BRASIL, 2018). Na Tabela 2, podem ser observadas as metas estabelecidas pelo INEP para cada município da bacia, no ano de 2017.

Conforme disposto na Figura 5, o IDEB nos anos iniciais do ensino fundamental atingiu as metas estabelecidas em nove municípios da bacia, apenas Aripuanã, que deveria ter atingido meta de 5,6 em 2017 ficou abaixo, com um valor de 5,4. Em contrapartida, nos anos finais do ensino fundamental sete municípios 
atingiram as metas, e três (Juína, Rondolândia e Vilhena) ficaram abaixo dos valores estabelecidos (4,8, 4,3 e 5,1 respectivamente), com valores de 4,7, 4,2 e 4,9, respectivamente.

Tabela 2: Metas para o IDEB nos anos iniciais e finais do ensino fundamental, nos municípios pertencentes à bacia hidrográfica do rio Roosevelt, ano de 2017.

\begin{tabular}{lll}
\hline Municípios & Anos iniciais & Anos finais \\
\hline Aripuanã & 5,6 & 4,7 \\
Cacoal & 5,6 & 5,1 \\
Colniza & 4,7 & 4,3 \\
Espigão do Oeste & 5,6 & 5,2 \\
Juína & 5,5 & 4,8 \\
Ministro Andreazza & 5,1 & 5,1 \\
Novo Aripuanã & 4,1 & 4,0 \\
Pimenta Bueno & 5,6 & 4,9 \\
Rondolândia & 3,8 & 4,3 \\
Vilhena & 5,6 & 5,1 \\
\hline
\end{tabular}

Fonte: INEP (2020).

Vale ressaltar também que nos municípios de Ministro Andreazza (6,9), Cacoal $(6,3)$ e Pimenta Bueno $(6,2)$ foi possível observar as maiores médias para os anos iniciais do ensino fundamental, enquanto nos anos finais do ensino fundamental se destacam Ministro Andreazza (5,2), Espigão d'Oeste $(5,2)$ e Cacoal $(5,1)$ (Figura 5).

No entanto, observa-se que as médias caem consideravelmente nos últimos anos do ensino fundamental se comparado aos anos iniciais (Figura 5). Corroborando com uma análise feita por Fernandes (2016), em que os resultados do IDEB no Brasil, de 2005 a 2013, em geral, foram mais significativos nos primeiros anos do ensino fundamental, e menores nos anos finais, tendo em vista que no primeiro caso também houve melhora de desempenho na prova Brasil.

Segundo Chirinéa et al. (2015), o IDEB é um importante parâmetro para avaliar e melhorar a qualidade da educação brasileira, no entanto não é o suficiente, uma vez que leva em conta apenas duas variáveis para o cálculo, desconsiderando outros fatores relevantes que interferem no desempenho dos estudantes, como: situação socioeconômica e cultural do aluno, formação e valorização dos professores, infraestrutura escolar, dentre outros.

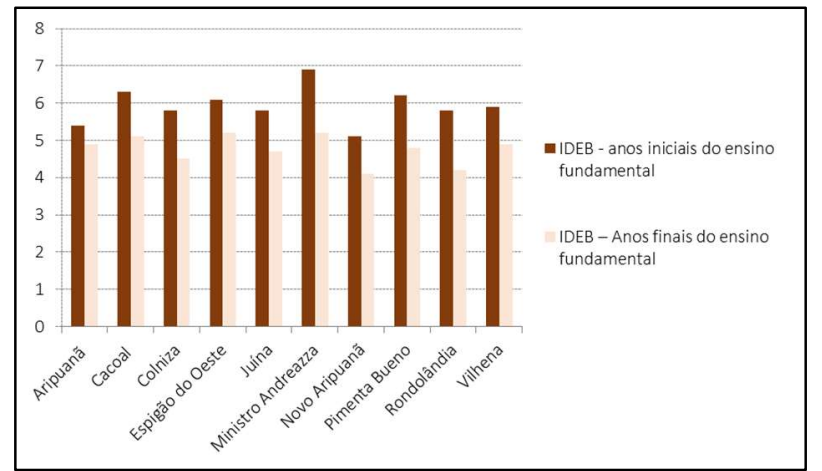

Figura 5: IDEB dos anos iniciais e finais do ensino fundamental (Rede Pública), nos municípios da bacia do rio Roosevelt, ano de 2017.

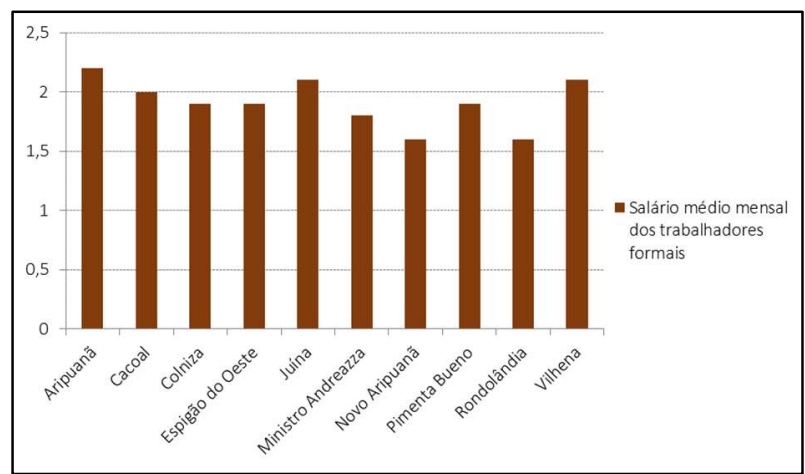

Figura 6: Salário médio mensal dos trabalhadores formais (Salário mínimo), dos municípios pertencentes à bacia hidrográfica do rio Roosevelt, no ano de 2018.

Outro aspecto a ser mencionado é que, a educação como um dos critérios utilizados para a medição do desenvolvimento de um município, pode ter relação com o fato de que em alguns municípios com maiores 
valores de IDEB, assim como taxas de escolarização altas, também são encontrados valores altos de IDH. Dentre os municípios citados, destaca-se Cacoal que apresenta valores de IDH corroborando com os indicadores de educação evidenciados.

Na Figura 6, observa-se que o salário médio mensal dos trabalhadores formais (salário mínimo), dos municípios pertencentes à bacia no ano de 2018 apresentou-se maior nos municípios de Aripuanã, Juína e Vilhena, ficando em torno de 2,2 para o primeiro e 2,1 para os outros dois. Por outro lado, Novo Aripuanã e Rondolândia tiveram os menores salários, em torno de 1,6.

Como indicador de estado e pressão escolhido para a bacia do rio Roosevelt tem-se a lavoura, pastagens, matas e florestas. Para este indicador foi analisada a proporção de estabelecimentos para cada uma dessas formas de uso do solo e obteve-se o gráfico representado em Figura 7.

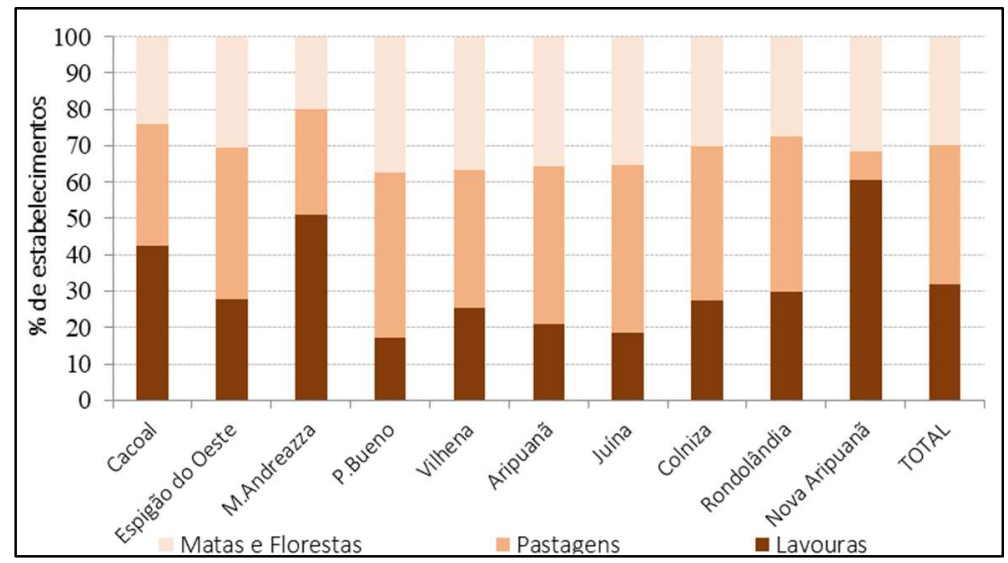

Figura 7: Proporção de estabelecimentos envolvida na produção de Lavouras, Pastagens, Matas e Florestas no ano de 2016 para os municípios que fazem parte da Bacia Hidrográfica do Rio Roosevelt.

Por meio deste indicador verificou-se que a utilização do solo na bacia apresenta-se equilibrada entre essas três formas selecionadas para análise, o valor que aponta para essa afirmação é a proporção obtida quando considerados todos os municípios, constata-se que $32,05 \%, 37,98 \%$ e $29,97 \%$ dos estabelecimentos de todos os municípios da bacia hidrográfica do rio Roosevelt, utilizam o solo para lavouras, pastagens e matas e florestas, respectivamente.

Em análise dos municípios por delimitação geográfica, observa-se a predominância das lavouras nos municípios pertencentes ao estado do Mato Grosso e para os municípios de Rondônia a predominância é de pastagens. $\mathrm{O}$ uso por lavouras e pastagens são importantes indicadores da qualidade ambiental, pois estes estão diretamente afetando o solo e a água e por serem sistemas implantados pela atividade antrópica, de não ocorrência natural na região exerce em muitos casos uma pressão sobre o meio ambiente (TUNDISI, 2011).

Em estudo desenvolvido por Carvalho et al. (2020), no município de Cacoal, identificou-se que a cobertura vegetal natural foi reduzida consideravelmente, ao longo do tempo, dando lugar ao uso do solo para pastagens que se destacou como o principal uso dentre as demais categorias, aumentando a pressão sobre os recursos naturais.

Por outro lado, as matas e florestas, possibilitam a conservação das propriedades naturais do meio, 
indicando o estado da Bacia Hidrográfica em estudo. Tendo em vista os indicadores de densidade populacional e as ações de incentivo do Governo desde o início da colonização dos estados, gerando uma interferência no uso e ocupação da Bacia do Rio Roosevelt, esperava-se que a proporção de uso para matas e florestas fosse menor, no entanto a Figura 7 comprovou o contrário.

Com relação aos indicadores econômicos, foi verificado o PIB dos municípios e constatado (conforme disposto na Figura 8) que o menor PIB é de $\mathrm{R} \$$ 6.757,82, em Novo Aripuanã, já o maior valor encontrado é de $R \$ 32.753,74$, no município de Aripuanã.

Em se tratando de indicador de impacto, consta em Figura 9, o gráfico com a ocorrência de internações por diarreia por mil habitantes de cada município da bacia.

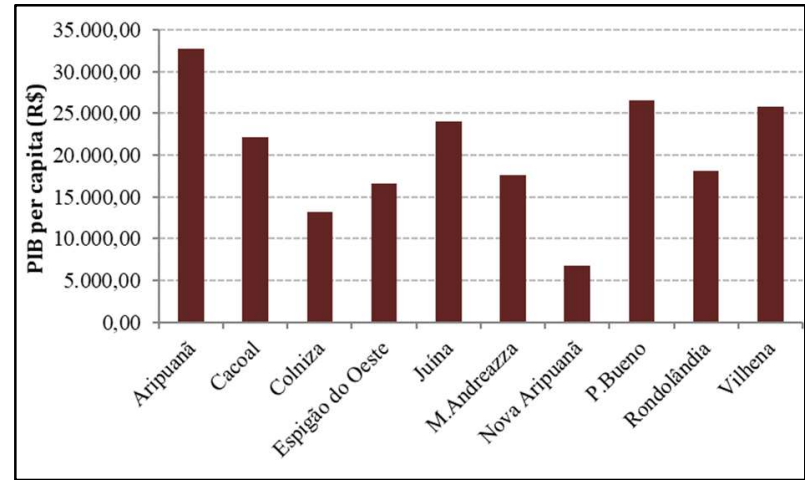

Figura 8: Produto Interno Bruto - PIB no ano de 2016 para os municípios pertencentes à bacia hidrográfica do Rio Roosevelt.

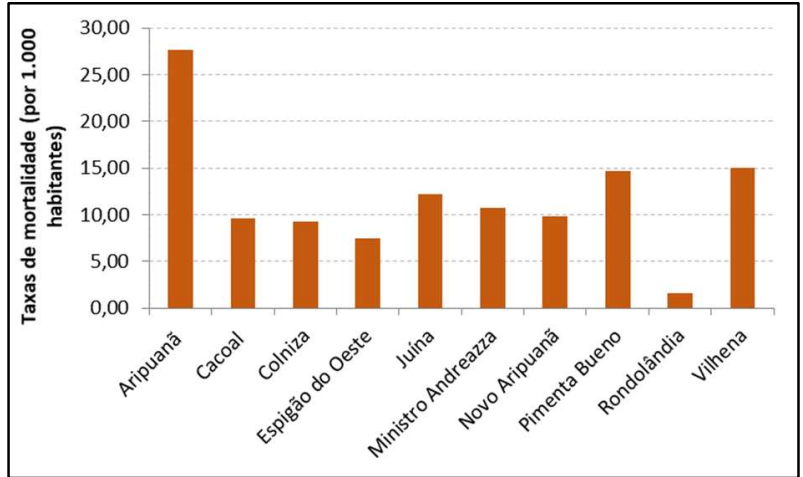

Figura 9: Casos de internações por diarreia por mil habitantes no ano de 2016 em cada município pertencente à bacia hidrográfica do rio Roosevelt.

Verificou-se que Pimenta Bueno (4,2), Espigão do Oeste (4) e Aripuaña $(3,6)$ apresentaram as maiores quantidades de internações por diarreia a cada 1.000 habitantes e os municípios que apresentaram os menores índices foram Colniza (0,5), Juína (1) e Cacoal (1). Para Rondolândia o dado referente a casos de internações por diarreia se encontra inexistente.

Ainda com interesse de verificar o impacto consultou-se a taxa de mortalidade infantil, dispostos na Figura 10. Com base nos dados é possível constatar que os municípios com maiores taxas de mortalidade infantil referem-se à Aripuaña $(27,62)$ Vilhena $(15)$, Pimenta Bueno $(14,63)$ e as menores taxas foram observadas em Rondolândia $(1,6)$, Espigão do Oeste $(7,5)$ e Colniza $(9,24)$.

Em se tratando deste indicador verifica-se que somente Rondolândia e Espigão do Oeste encontramse dentro da meta 3.2 dos Objetivos do Desenvolvimento Sustentável - ODS para o Brasil, de até 2030 reduzir a mortalidade infantil para no máximo 8 óbitos por 1000 nascidos vivos (IPEA, 2020), porém deve ser considerado que o intervalo para alcançar essa meta é de 10 anos havendo espaço suficiente para os demais municípios identificarem as causas da elevada mortalidade e atuarem para a resolução desse problema.

Observando os dados de esgotamento sanitário, representados na Figura 11, este pode ser um agente causador da elevada taxa de mortalidade infantil observada nos municípios da bacia hidrográfica em estudo. Verificou-se que somente Cacoal possui mais de $50 \%$ da população com esgotamento sanitário adequado. Os municípios com maiores proporções deste serviço sendo oferecido de forma adequada são Cacoal (52,5\%) e Pimenta Bueno (37,9\%). 
Ressalta-se que para este indicador os municípios podem ter alcançado melhorias, já que o índice nacional apresentou um acréscimo em 2019 quando comparado aos três últimos anos analisados, passando a apresentar $68,3 \%$ de domicílios com esgotamento sanitário adequado, apesar desse acréscimo observa-se que ainda há uma situação precária na oferta, tendo em vista a dimensão do país e o elevado número de residências que não são atendidas pelos serviços de coleta e tratamento de esgoto (IBGE, 2020).

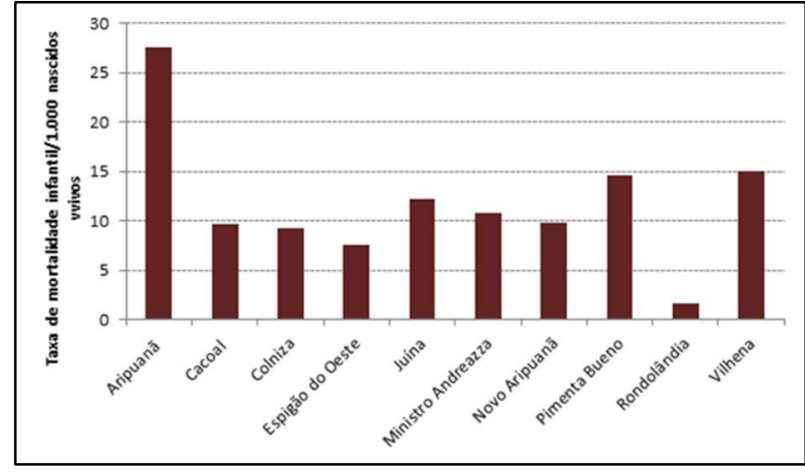

Figura 10: Taxa de mortalidade infantil (óbitos por 1000 nascidos vivos), nos municípios da bacia do rio Roosevelt, ano de 2017.

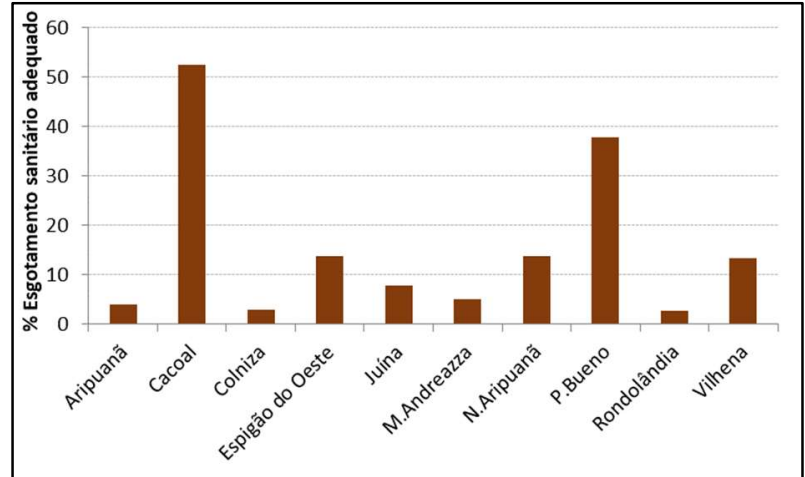

Figura 11: Porcentagem de domicílios com esgotamento sanitário adequado no ano de 2010, para os municípios pertencentes à bacia hidrográfica do rio Roosevelt.

Com relação ao capital financeiro e humano, Toigo et al. (2016) concluíram que a falta proporciona um caminho oposto não somente ao desenvolvimento humano e econômico, mas também ao sustentável mesmo sendo gerada menor pressão na natureza, devido à baixa eficiência das políticas ambientais.

O município com menor valor de PIB (Nova Aripuanã) observado na bacia hidrográfica, dados apresentados em Figura 8, está na posição 5114 de 5570 municípios, e o município com maior valor de PIB (Aripuanã) está na posição 886, indicando que a bacia hidrográfica sofre interferência de variadas realidades econômicas, o que pode influenciar no desenvolvimento dos cuidados com a qualidade ambiental, assim como com a intensidade das pressões aplicadas em toda extensão da bacia.

Para este indicador observa-se que, apesar do município que apresentou o menor PIB não ser o município a apresentar o maior número de internações por diarreia, e tendo se destacado Aripuanã com o terceiro maior número de internações por mil habitantes, é necessário avaliar outros indicadores para identificar o que tem determinado a ocorrência de diarreias nos municípios analisados.

Outro fator que justifica a necessidade de averiguar o que tem influenciado a ocorrência de diarreias é o fato dos municípios que apresentaram as menores taxas de internações (Colniza e Juína) são os que possuem a menor porcentagem de esgotamento sanitário adequado.

\section{CONCLUSÕES}

O banco de dados do IBGE-Cidades@ possibilita uma análise de indicadores aplicáveis em territórios fluviais onde se almeja uma gestão por meio de Comitês de Bacia, dos municípios que os contém ou de uma proposta de governança por atores hidrográficos que buscam a garantia de uma justiça ambiental.

Com a técnica de análise de dados estatísticos RAE somada ao método de análise e agrupamento de indicadores proposto pelo PEIR foi possível discutir os dados representativos dos municípios pertencentes 
em partes ou totalmente à Bacia Hidrográfica do Rio Roosevelt, um território fluvial que envolve três estados Pan-Amazônicos.

Por meio da pesquisa realizada, com os subsídios técnicos e metodológicos aplicados constata-se que a bacia sofre diferentes pressões ao longo de sua área, relacionadas ao aumento populacional, uso do solo, economia e esgotamento sanitário. E, além disso, em alguns municípios, como Cacoal e Vilhena, foi possível observar uma relação entre indicadores como IDH, PIB, IDEB e taxa de escolarização.

Os indicadores analisados, ainda que preliminares, evidenciam a necessidade de aprofundamento nos estudos sobre os mesmos para se subsidiarem diálogos em prol de uma implantação de gestão neste território fluvial, alinhando diretrizes entre os estados Pan-Amazônicos que integram essa unidade de gestão. Tendo como fio condutor a urgência de um diálogo hídrico compartilhado entre todos os atores representativos. Do contrário corre-se o risco dos dados apresentados pelo IBGE seguirem revelando uma ausência de comprometimento e da justiça ambiental aos povos que integram esse espaço.

AGRADECIMENTOS: O presente trabalho foi realizado com apoio da Coordenação de Aperfeiçoamento de Pessoal de Nível Superior - Brasil (CAPES) - Código de Financiamento 001, agradecemos também ao Programa de Mestrado Profissional em Rede Nacional em Gestão e Regulação de Recursos Hídricos - Prof. Água, Projeto CAPES/ANA AUXPE №. 2717/2015, pelo apoio científico aportado até o momento.

\section{REFERÊNCIAS}

BARRETO, A. M.. Território de Águas na Amazônia:

Ribeirinhos e o Direito à Coletiva da Terra. Juruá, 2019.

BRASIL. Lei n.9.433, de 08 de janeiro de 1997. Institui a Política Nacional de Recursos Hídricos, cria o Sistema Nacional de Gerenciamento de Recursos Hídricos, regulamenta o inciso XIX do art. 21 da Constituição Federal, e altera o art. 10 da Lei no 8.001, de 13 de março de 1990, que modificou a Lei no 7.990, de 28 de dezembro de 1989. Presidência da República. Casa Civil. Subchefia para Assuntos Jurídicos. Brasília: DOU, 1997.

BRASIL. Ministério da Educação. IDEB: Apresentação. Brasília: MEC, 2018.

CARAMELLO, N.; PINHEIRO, Z. C.; LIMA, L. F. M.. Análise Socioambiental integrada de bacia hidrográfica através da metodologia PEIR: estudo de caso da Bacia do Igarapé D’Alincurt. In: Amazônia: recursos hídricos e diálogos socioambientais. 2011

CARAMELLO, N.; SAURI PUJOL, D.. El Río: Un Protagonista Oculto En El Diálogo De Las Aguas. Mercator (Fortaleza), Fortaleza, v.15, n.3, p.107-126, 2016. DOI: http://dx.doi.org/10.4215/RM2016.1503.0007

CARVALHO, M. B. F.; NEIMOG, W. S.; MOREIRA, M. F.; CAMPOS, D. F.; SOUZA, N. F. C.; NASCIMENTO, J. F.; CARAMELLO, N.. Análise do uso e cobertura do solo no município de Cacoal, Rondônia nos anos de 1985 e 2019. In: SIMPÓSIO POTIGUAR DE PÓS-GRADUAÇÃO EM CIÊNCIAS FLORESTAIS, 2. Anais. Macaíba: UFRN, 2020.
CHIRINÉA, A. M.; BRANDÃO, C. F.. O IDEB como política de regulação do Estado e legitimação da qualidade: em busca de significados. Ensaio: Avaliação e Políticas Públicas em Educação, Rio de Janeiro, v.23, n.87, p.461-484, 2015.

COTTA, T.; SILVA, F.; GONÇALVES, A.; LIMA, D.; CARAMELLO, N.. Indicadores socioambientais como instrumento de gestão de território fluvial: comunidade de Rolim de Moura do Guaporé-RO. Revista de geografia e Ordenamento do Território (GOT), Coimbra, n.17, p.29-54, 2019. DOI: http://dx.doi.org/10.17127/got/2019.17.002

IBGE. Instituto Brasileiro de Geografia e Estatística. Estatísticas: Cidade e estados. Rio de Janeiro: IBGE, 2020.

IBGE. Instituto Brasileiro de Geografia e Estatística. Agência IBGE: Noticias: Características dos domicílios. Rio de Janeiro: IBGE, 2020

IBGE. Instituto Brasileiro de Geografia e Estatística. Painel de Indicadores. Indicadores Sociais: escolarização. Rio de Janeiro: IBGE, 2020.

IBGE. Instituto Brasileiro de Geografia e Estatística. Censo 2010: escolaridade e rendimento aumentam e cai mortalidade infantil. Rio de Janeiro: IBGE, 2010.

IPEA. Instituto de Pesquisa Econômica Aplicada. Objetivos de Desenvolvimento Sustentável: ODS - Meta 3. Saúde e Bem-estar. 2020.

IDARON. Agência de Defesa Sanitária Agrosilvopastoril do Estado de Rondônia. Relatório de Gestão: Relatório de 
Atividades IDARON 2018. Porto Velho: IDARON, 2018.

INEP. Instituto Nacional de Estudos e Pesquisas Educacionais Anísio Teixeira. Índice de Desenvolvimento da Educação Básica: Resultados e Metas. Brasília: Copyright MEC, 2020

FERNANDES, R.. A universalização da avaliação e a criação do IDEB: pressupostos e perspectivas. Em Aberto, Brasília, v.29, n.96, p.99-111, 2016. DOI: http://dx.doi.org/10.24109/21766673.emaberto.29i96.\%25p

NOLAN, D.; SPEED, T.. Stat labs: mathematical statistics through applications. New York: Springer-Verlag, 2000.

NUNES, A.; MOREIRA, C. O.; PAIVA, I. R.; CUNHA, L. S.. Territórios de Água. Coimbra: Centro de estudos de Geografia e do reordenamento do território - CEGOT, 2016.

PNUMA. Programa das Nações Unidas para o Meio Ambiente. Metodologia para elaboração de Informes GEO Cidades: manual de aplicação. Rio de Janeiro: PNUMA, 2002.

PNUD; IPEA; FJP. Programa das Nações Unidas para o Desenvolvimento; Instituto de Pesquisa Econômica Aplicada; Fundação João Pinheiro. $O$ índice de desenvolvimento humano municipal Brasileiro: Série Atlas do Desenvolvimento Humano no Brasil 2013. Brasília: PNUD, 2013.

PORTO, M. F. A.; PORTO, R. L.. Gestão de Bacias Hidrográficas. Estudos Avançados, São Paulo, v.22, n.63, p.44-60, 2008.

RIBEIRO, P. N. T.. Variáveis indicadoras de qualidade ambiental na assembleia de peixes de um trecho do Rio Roosevelt na Região Amazônica. Dissertação (Mestrado em Ciências Ambientais) -Universidade Federal do Amazonas, Humaitá, 2017.

RONDÔNIA. Lei Complementar n.255, de 25 de janeiro de 2002. Institui a Política, cria o Sistema de Gerenciamento e o Fundo de Recursos Hídricos do Estado de Rondônia e dá outras providências. Porto Velho: DOE, 2002.
SCARPIN, J. E.; SLOMSKI, V.. Estudo dos fatores condicionantes do índice de desenvolvimento humano nos municípios do estado do Paraná: instrumento de controladoria para a tomada de decisões na gestão governamental. Revista de Administração Pública - RAP, Rio de Janeiro, v.41, n.5, 2007.

SANTOS, A. M.; HOLMES, D. C. S.; RAMOS, H. F.. Densidade demográfica: um estudo comparativo de duas metodologias a partir de imagens orbital e sub-orbital na cidade de Aparecida de Goiânia/Goiás. Ateliê Geográfico, Goiânia, v.12, n.1, p.175-200, 2018.

SILVA, S. S. F.; CÂNDIDO, A. C.; RAMALHO, C. A. M.; SILVA, S. L. R.; SANTANA, G. P.. Diagnóstico situacional dos resíduos sólidos urbanos no município De Cuité-PB: uma aplicação do sistema de indicador de sustentabilidade Pressão - Estado Impacto - Resposta (P-E-I-R). Revista Gestão Industrial, v.08, n.03, p.72-90, 2012.

SILVA, F. M.; MEDEIROS, P. M. S.; CARAMELLO, N. D. A.. Alternativas para melhoria da Gestão Hídrica na Bacia Hidrográfica Ribeirão Cacau em Alvorada D'Oeste. Revista Ibero-Americana de Ciências Ambientais, v.11 n.6, p.492509, 2020. DOI: https://doi.org/10.6008/CBPC2179$\underline{6858.2020 .006 .0040}$

SILVA, R. G. C.. Globalização, agricultura e a formação do meio técnico-científico informacional em Rondônia. ACTA Geográfica, Boa Vista, v.7, n.15, p.69-83, 2013. DOI: http://dx.doi.org/10.5654/acta.v7i15.1383

TUNDISI, J. G.; TUNDISI, T. M.. Recursos Hídricos no Século XXI. São Paulo: Oficina de Textos, 2011.

TOIGO, C. H.; MATTOS, E. J.. Desenvolvimento e meio ambiente: o que os principais índices têm a revelar?. Ensaios FEE, v.37, n.2, p.553-580, 2016

UNICEF. Fundo das Nações Unidas para a Infância. Enfrentamento da exclusão escolar no Brasil. Brasília: Cross Content, 2014.

A CBPC - Companhia Brasileira de Produção Científica (CNPJ: 11.221.422/0001-03) detém os direitos materiais desta publicação. Os direitos referem-se à publicação do trabalho em qualquer parte do mundo, incluindo os direitos às renovações, expansões e disseminações da contribuição, bem como outros direitos subsidiários. Todos os trabalhos publicados eletronicamente poderão posteriormente ser publicados em coletâneas impressas sob coordenação da Sustenere Publishing, da Companhia Brasileira de Produção Científica e seus parceiros autorizados. Os (as) autores (as) preservam os direitos autorais, mas não têm permissão para a publicação da contribuição em outro meio, impresso ou digital, em português ou em tradução. 